\title{
Gabapentin as an adjuvant treatment in renal colic: A randomized double-blind clinical trial
}

\author{
Goodarzi D. ${ }^{1 *}$, Cyrus A. ${ }^{1}$, Baghinia M. R. ${ }^{1}$, Sameni D. ${ }^{2}$ \\ ${ }^{1}$ Department of surgery, Arak University of Medical Science. Arak, Iran. \\ ${ }^{2}$ Graduate of General Physician, Arak University of Medical Sciences. Arak, Iran.
}

Accepted 10 June, 2013

\begin{abstract}
Acute renal colic probably is the most painful event a person can endure and pain relief with minimal side effects is a humane approach. Gabapentin is an antiepileptic drug, also used in the treatment of neuropathic pain but it's efficacy and safety in treating renal colic has not been demonstrated. We studied patients with renal colic to assess the efficacy and safety of single dose of $600 \mathrm{mg}$ oral Gabapentin compared to placebo as an analgesic in renal colic. In this randomized double-blind controlled clinical trial, 100 patients aged 20-40 y/o with renal colic, admitted to Vali Asr hospital of Arak city, Iran, were randomized to receive $0.5 \mathrm{mg} / \mathrm{kg}$ of Pethidin hydrochloride and single-dose of $600 \mathrm{mg}$ Gabapentin $(n=50)$ or Pethidin hydrochloride $0.5 \mathrm{mg} / \mathrm{kg}$ and placebo $(n=50)$. Clinical signs and symptoms, abdominal pain intensity (based on visual analog pain score (VAS)) were recorded prior to, and 1,2,3,6 $\mathrm{h}$ after drug administration and compared between groups. The significant pain score reduction was reported in Gabapentin group $(p<0.001)$. Nausea severity score, mean vomiting frequency and additional Pethidin hydrochloride administration after intervention in Gabapentin group was significantly lower than placebo group $(p<0.001)$. According to the result of present study, the use of Gabapentin can effectively reduce the pain intensity, nausea, vomiting and the need of analgesics among patients with renal colic.
\end{abstract}

Key words: Abdominal pain, gabapentin, pain, renal colic.

\section{INTRODUCTION}

Acute renal colic probably is the most painful event a person can endure. Renal colic affects approximately 1.2 million people each year, and accounts for approximately $1 \%$ of all hospital admissions (Wolf and Howes, 2012). Pain relief is one of the most essential aspects of the successful management of renal colic. In recent years, great emphasis has been laid on ensuring that adequate pain relief is coupled with minimal side effects (Helfand and Freeman, 2009).

Non-steroidal anti-inflammatory drugs (NSAIDs) and opioids have been used as a pain relief in renal colic (Curry and Kelly, 1995; Smally, 1997). Opioids have some advantages such as cheapness, titratability and potency but there are concerns of dependency and drug seeking behavior presenting as renal colic, which may limit their usefulness (Reich and Hanno, 1997). NSAIDs act directly on the main cause of pain; prostaglandin release, and have been shown to be effective, particularly when used intravenously (Tramer et al., 1998) but NSAIDS are generally not titratable with some severe adverse effects including renal failure and gastrointestinal bleeding and may be less immediate and potent in their action.

Gabapentin as an antiepileptic drug is used for epilepsy, neurodegenerative disorders, and pain (Vedula et al., 2011; Moore et al., 2011). A number of animal studies

*Corresponding author. E-mail: dgoudarzi@arakmu.ac.ir. Tel/Fax: +988612221041 
and clinical trials have shown that compounds related to Gabapentin are useful for treating neuropathic pain (Rosner et al., 1996; Segal et al., 1996; Khan et al., 1998; Solaro et al., 1998). Placebo-controlled clinical trials also have indicated a therapeutic role of Gabapentin in diabetic neuropathy (Backonja et al., 1998; Seidl et al., 1999). Some recent publications also have used Gabapentin as an analgesic in established acute postoperative pain (Straube et al., 2010), implicating even broader use of Gabapentin in variety of pain states. There are some non-controlled, open-labeled clinical studies supporting the of Gabapentin role in reducing spontaneous pain and allodynia regardless of the etiology (Mao et al., 2000), so the mechanism of Gabapentin effect on pain control is not clear (Mao et al., 2000). The aim of present study was to assess the efficacy and safety of single dose of $600 \mathrm{mg}$ oral Gabapentin compared with placebo as an adjuvant analgesic in renal colic.

\section{MATERIALS AND METHODS}

In this randomized double-blind controlled clinical trial, 100 patients' aged 20-40 years old with renal colic, admitted to Vali Asr hospital of Arak city, were enrolled in the study. Simple randomization was applied based on the patients' code (even: control, odd: treatment). Exclusion criteria were: Cardio-vascular disease or hypertension, analgesic consumption or psychotropic medication, epilepsy, chronic vertigo, ataxia and pregnancy or breast feeding.

This study was ethically approved by the research ethical committee of the Arak University of medical Sciences, Iran. Written informed consent was obtained from all patients or their relatives before the trial. Due to the lack of availability of intravenous diclofenac sodium in our country, Iran, Pethidin hydrochloride is routinely used for pain management in renal colic.

All of the enrolled patients were randomized to receive $0.5 \mathrm{mg} / \mathrm{kg}$ of Pethidin hydrochloride and single-dose of $600 \mathrm{mg}$ Gabapentin $(n=50)$ or $0.5 \mathrm{mg} / \mathrm{kg}$ Pethidin hydrochloride and placebo $(n=50)$. The patients and clinicians who were responsible for visiting the patients remained blind during the trial. Clinical symptoms, physical signs including nausea and vomiting and pain intensity (based on visual analog pain score (VAS)) were recorded prior to and 1, 2, 3 and $6 \mathrm{~h}$ after drug administration. Nausea severity (was scored 0 3 ), frequency of vomiting and drug adverse effects including headache, vertigo and sleepiness were recorded 1,2, 3 and $6 \mathrm{~h}$ after intervention too. Additional Pethidin hydrochloride was administered based on patients' request and recorded. All statistical analysis were performed with the Statistical Package for the Social Sciences (SPSS) software 16 (t-test, paired t-test, chisquare) and statistical significance was considered at $P \leq 0.05$.

\section{RESULTS AND DISCUSSION}

In this study, there were a total of 100 patients with renal colic. The mean age of studied population was $32.31( \pm 5.87)$ years old and $71 \%$ of them were men. There was no significant difference between two groups regarding to age, gender, weight $(p>0.05$, Table 1$)$ and initial clinical evaluation and VAS score (Table 2). The VAS scores upon admission to the hospital and after intervention were measured and is shown in Table 2. The significant pain scores reduction was reported in Gabapentin group $(p<0.001)$ and the difference between VAS score of two groups increased during the hours.

Nausea severity score in first evaluation in Gabapentin group was $2.68 \pm 0.68$ vs. $2.74 \pm 0.44$ in the control group (P: NS) meanwhile after drug administration, Gabapentin group was significantly lower than placebo group $(6.36 \pm 3.16$ vs. $7.68 \pm 2.03$, respectively). Mean vomiting frequency in intervention and control group were $0.00 \mathrm{vs.}$ $0.38 \pm 0.77$ which was respectively, and significantly different $(p<0.001)$. Mean additional patients' requested Pethidine hydrochloride in Gabapentin group was $0.8 \pm 0.23 \mathrm{mg} / \mathrm{kg}$ which was significantly less than control group $(1.11 \pm 0.42 \mathrm{mg} / \mathrm{kg})$. Dizziness as a drug adverse effect was seen in $3(6 \%)$ patients in Gabapentin group compared with control group which was significantly different $(P<0.001)$. Any other adverse effect was not seen in both treatment and control group.

Based on the results of present study, Gabapentin can effectively reduce the pain intensity, nausea, vomiting and the need of analgesics among patients with renal colic. According to Mao et al. (2000) Gabapentin is effective in reducing neuropathic pain associated with diabetic neuropathy, post herpetic neuralgia, multiple sclerosis and cancer-related neuropathic pain.

Several mechanisms such as modulation of gammaaminobutyric acid (GABA) synthesis, peripheral effect on abnormal sodium channel activation and a specific binding site (the $\alpha_{2} \delta$ subunit of voltage dependent calcium channels), are considered for Gabapentin analgesic effect but there is little information to support these hypotheses (Mao et al., 2000). Angello (2005) noticed GABA analogs work both peripherally and centrally to relieve pain. GABA analogs have interactions with calcium channels which are considered having potential role in renal colic. Calcium channel antagonists like nifedipine have vasodilatory effect on ureter smooth muscles and can relieve the pain and help passage of the renal calculi. Based on this possible central and peripheral interference with pain pathways and potential ureter smooth muscle relaxation, Gabapentin can provides superior pain relief for renal colic but optimal dose and how to use of Gabapentin is unclear yet (Imani, 2012).

Straube (2010) showed that $250 \mathrm{mg}$ Gabapentin is significantly effective than placebo in postoperative pain relief in participants having pain following dental or orthopedic surgery but the clinical value of it is limited and inferior to commonly used analgesics. There are some clinical trials which has been shown the suppressant effect of Gabapentin in postoperative nausea and vomiting (Pandey, 2006), chemotherapy induced nausea (Guttuso et al., 2003) and some other medical situations. In the present study, the nausea severity score and the mean vomiting frequency in Gabapentin group was less than control group. In patients with renal colic, the nausea and vomiting is due to severe pain (Kehlet et al, 2003), so it seems that 
Table 1. Baseline characteristics upon admission to the hospital.

\begin{tabular}{lcc}
\hline Parameter & Gabapentin Group & Control Group \\
\hline Age(years) & $68.18 \pm 9.26$ & $66.98 \pm 7.62$ \\
Weight(kg) & $71.62 \pm 10.30$ & $69.92 \pm 7.98$ \\
Male/Female(n) & $36 / 14$ & $35 / 15$ \\
Opacity in KUB(n) & 32 & 38 \\
\hline
\end{tabular}

Table 2. Abdominal pain scores (VAS).

\begin{tabular}{lccc}
\hline Groups/ Time & Gabapentin & Control(placebo) & P value \\
\hline First evaluation & $9.32 \pm 0.79$ & $9.28 \pm 0.8$ & 0.804 \\
Hour 1 & $7.66 \pm 1.13$ & $8.76 \pm 0.93$ & $<0.001^{*}$ \\
Hour 2 & $5.82 \pm 1.62$ & $7.94 \pm 0.01$ & $<0.001^{*}$ \\
Hour 3 & $4.02 \pm 2.12$ & $7.24 \pm 1.11$ & $<0.001^{*}$ \\
Mean pain score & $2.36 \pm 1.83$ & $5.88 \pm 1.11$ & $<0.001^{*}$ \\
\hline
\end{tabular}

${ }^{*} p<0.05$, statistically significant.

effective pain relief can reduce the frequency and intensity of nausea and vomiting. In this double blind placebocontrolled clinical trial, we used Gabapentin as an adjuvant analgesic and it demonstrated that administration of $600 \mathrm{mg}$ Gabapentin reduced the intensity of pain among patients with renal colic compared to control group without any severe side effects $(p<0.001)$. Gabapentin has few interactions with major classes of drugs since it is not metabolized in the body. It seems that the nontoxic nature of Gabapentin and its suitable analgesic effects in renal colic proposes the drug as an adjuvant treatment in renal colic.

\section{ABBREVIATIONS}

NSAIDs, Non-steroidal anti-inflammatory drugs; VAS, visual analog pain score; GABA, gamma-aminobutyric acid.

\section{REFERENCES}

Angello JT (2005). The treatment of renal colic with gaba analogs. Patent: EP1094804. Available from: http://www.freepatentsonline.com/EP1094804B1.html.

Backonja M, Beydoun A, Edwards KR (1998). Gabapentin for the treatment of post herpetic neuralgia a randomized controlled trial. JAMA. 280:1837-42.

Guttuso TJR, Roscoe J, Griggs J (2003). Effect of gabapentin on nausea induced by chemotherapy in patients with breast cancer. THE LANCET. 361(9370):1703-1705.

Imani F, Rahimzadeh P (2012). Gabapentinoids: Gabapentin and pregabalin for postoperative pain management. Anesth Pain. 2(2):523.

Kehlet H, Dahl JB (2003). Anesthesia, surgery, and challenges in postoperative recovery. The Lancet. 362:1921-28.
Khan OA (1998). Gabapentin relives trigeminal neuralgia in multiple sclerosis patients. Neurol. 51:611-4.

Mao J, Chen LL (2000). Gabapentin in Pain Management. Anesth. Analg. 91:680-7.

Moore RA, Wiffen PJ, Derry S, McQuay HJ (2011). Gabapentin for chronic neuropathic pain and fibromyalgia in adults. Cochrane database of systematic reviews (Online) (3):CD007938.

Pandey CK, Priye S, Ambesh SP, Singh S, Singh U, Singh PK (2006). Prophylactic gabapentin for prevention of postoperative nausea and vomiting in patients undergoing laparoscopic cholecystectomy: A randomized, double-blind, placebo-controlled study. J. Postgrad. Med. 52:97-100.

Segal AZ, Rordorf G (1996). Gbapentin as a novel treatment for postherpetic neuralgia. Neurol. 46:1175-6.

Seidl JJ, Slawson JG (1999). Gabapentin for painful diabetic neuropathy. J. Fam Pract. 48:173-4.

Solaro C, Lunardi GL, Capello E, Inglese M, Uccelli MM,Uccelli A, Mancardi GL (1998). An open-label trial of Gabapentin treatment of paroxysmal syndromes in multiple sclerosis patients. Neurol. 51:60911.

Straube S, Derry S, Moore RA, Wiffen PJ, McQuay HJ (2010). Single dose oral Gabapentin for established acute postoperative pain in adults. Cochrane Database of Systematic Reviews. (5):CD008183.

Vedula SS, Bero L, Scherer RW, Dickersin K (2009). Outcome reporting in industry-sponsored trials of Gabapentin for off-label use. NEJM. 361(20):1963-71.

Wolf JS, Howes D, Craig S, Leslie SW, Siner RH (2012). Nephrolithiasis. http://emedicine.medscape.com/article/437096-overview. 